\title{
A CLASS OF REPRESENTATIONS OF THE FULL LINEAR GROUP. II
}

\author{
BY
}

\section{STEPHEN PIERCE( $\left.{ }^{1}\right)$}

ABSTRACT. Let $V$ be an $n$ odimensional vector space over complex numbers $C$. Let $W$ be the $m$ th tensor product of $V$. If $T \in \operatorname{Hom}_{C}(V, V)$, let $\otimes^{m} T \in \operatorname{Hom}_{C}(W, W)$ be the $m$ th tensor product of $T$. The homomorphism $T \rightarrow \otimes^{m} T$ is a representation of the full linear group $\mathrm{GL}_{n}(C)$. If $H$ is a subgroup of the symmetric group $S_{m}$, and $\chi$ a linear character on $H$, let $V_{\chi}^{m}(G)$ be the subspace of $W$ consisting of all tensors symmetric with respect to $H$ and $\chi$. Then $V_{\chi}^{m}(H)$ is invariant under $\otimes^{m} T$. Let $K(T)$ be the restriction of $\otimes^{m} T$ to $V_{\chi}^{m}(H)$. For $n$ large compared with $m$ and for $H$ transitive, we determine all cases when the representation $T$ $\rightarrow K(T)$ is irreducible.

I. Introduction. Let $V$ be an $n$-dimensional vector space over the complex numbers $C$, and let $W=\bigotimes^{m} V$ be the $m$ th tensor product of $V$. If $T \in \operatorname{Hom}_{C}(V, V)$, the map $T \rightarrow \bigotimes^{m} T$, the $m$ th Kronecker product of $T$, is a representation of the full linear group $\mathrm{GL}_{n}(C)$. Weyl [10] has shown that the irreducible components of this representation are in 1-1 correspondence with the irreducible representations of the symmetric group $S_{m}$. During the last decade, Marcus, Minc, Newman and others (e.g., [3], [4], [5], [6]) have studied certain subspaces of $W$ invariant under $\otimes^{m} T$ for the purpose of obtaining inequalities involving Schur functionsa class of matrix functions which includes the determinant and permanent. One notable result [6] was the proof of the Van der Waerden conjecture for positive semidefinite doubly stochastic matrices. Marcus has suggested the problem of determining all such subspaces which are irreducible invariant subspaces of $T \rightarrow$ $\bigotimes^{m} T$.

We assume some familiarity with Young's tableaus [1] and with the concept of blocks with respect to transitive permutation groups [18], [11].

II. Statement of results. Let $H$ be a subgroup of $S_{m}$ of order $b$ and $\chi$ an irreducible character of degree $r$ on $H$. For each $\sigma \in S_{m}$, let $P(\sigma) \in \operatorname{Hom}_{C}(W, W)$ be defined by

$$
P(\sigma) v_{1} \otimes \cdots \otimes v_{m}=v_{\sigma^{-1}(1)} \otimes \cdots \otimes v_{\sigma^{-1}(m)} .
$$

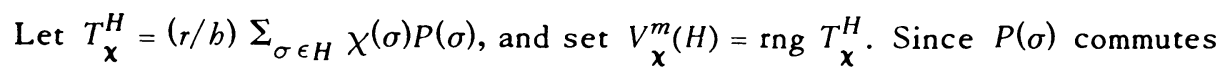

Received by the editors October 26, 1971.

AMS (MOS) subject classifications (1969). Primary 1580; Secondary 2020, 2085.

Key words and phrases. Young tableaus, symmetry class of tensors, permutation groups.

(1) Part of this work was supported by National Research Council of Canada grant A7862. 
with $\bigotimes^{m} T, V_{\boldsymbol{\chi}}^{m}(H)$ is invariant under $\bigotimes^{m} T$ for all $T \in \operatorname{Hom}_{C}(V, V)$. Let $K(T)$ be the restriction of $\bigotimes^{m} T$ to $V_{\chi}^{m}(H)$; then $T \rightarrow K(T)$ is a representation of $\mathrm{GL}_{n}(C)$. For example, if $H=S_{m}$ and $\chi \equiv 1, V_{\chi}^{m}(H)$ is the completely symmetric tensors and $K(T)=P_{m}(T)$, the $m$ th power transform. If $H=S_{m}$ and $\chi=\epsilon$, the alternating character, then $V_{\mathbf{x}}^{m}(H)=\bigwedge^{m} V$, the $m$ th Grassmann space, and $K(T)=C_{m}(T)$, the $m$ th compound transformation.

Clearly, $K(T)$ depends on four parameters $(m, n, H, \chi)$. We wish to determine for which of these quadruples $K(T)$ is irreducible.

In [7], Merris and the author proved that if $\chi$ is not linear, $K(T)$ always reduces. (In a recent communication, $R$. Freese gave a much simplified proof.) Thus we assume $\chi$ is linear. For the remainder of the paper, we also assume $H$ is transitive and $n$ sufficiently large compared with $m$. I believe, however, that no restrictions are necessary on $H$, and that we need only assume that $m \leq n$.

Theorem. Under the above restrictions, the only quadruples $(m, n, H, \chi)$ for which $K(T)$ is irreducible are

(a) $\left(m, n, S_{m}, \chi \equiv 1\right)$,

(b) $\left(m, n, S_{m}, X=\epsilon\right)$,

(c) $\left(3, n, A_{3}, \chi \equiv 1\right)$,

(d) $\left(4, n, A_{4}, \chi \equiv 1\right)$,

(e) $(4, n, H, \chi)$,

where, in (e), $H$ is a 2-Sylow subgroup of $S_{4}$, and $\chi$ is one of two possibilities: $H=\langle(12),(13)(24)\rangle$ and $\chi$ is defined by $\chi((12))= \pm 1, \chi((13)(24))=-1$.

III. Proof of the Theorem. Suppose $K(T)$ is irreducible.

Lemma 1. If $m \geq 5$, and $H=S_{m}$ or $A_{m}$, then $K(T)$ is in class (a) or (b) of the Theorem.

Proof. Since $A_{m}$ is simple for $m \geq 5$, the only linear character is the trivial one. If $m \leq n$, it is easy to show that the completely symmetric tensors are a proper

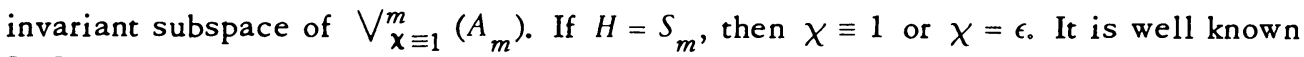
[10] that both of these yield irreducible representations of $\mathrm{GL}_{n}(C)$.

We will first solve the problem for $m \leq 4$ by direct computation. Then for $m \geq$ 5, we will force $H=A_{m}$ or $S_{m}$ and apply Lemma 1 .

If $K(T)$ is irreducible, it corresponds by Weyl's result [10] to a certain Young tableau $Y$. If $R$ is the row group and $Q$ the column group of $Y$, we form the Young symmetrizer $\Sigma_{\sigma \in R ; \tau \in Q} \epsilon(\tau) \tau \sigma$. The map $\sigma \rightarrow P(\sigma)$ is a homorphism of the group ring of $S_{m}$ into $\operatorname{Hom}_{C}(W, W)$, and yields an operator whose range is an irreducible invariant subspace of $\otimes^{m} T$. Thus the rank of this operator must equal the rank of $T_{\mathbf{x}}^{H}$. Let $d$ be the degree of the representation of $S_{m}$ corresponding to $Y$. Then $S=(d / m !) \Sigma_{\sigma \in R ; \tau \in Q} \epsilon(\tau) P(\tau \sigma)$ is idempotent. It follows [12] that the rank of $S$ is 
$(d / m !) \Sigma_{\sigma \in R ; \tau \in Q} \epsilon(\tau) n^{c(\tau \sigma)}$, where $c(\tau \sigma)$ is the number of orbits in $\tau \sigma$. Since $T_{\boldsymbol{\chi}}^{H}$ is also idempotent, we have

$$
\frac{d}{m !} \sum_{\sigma \in R ; \tau \in Q} \epsilon(\tau) n^{c(\tau \sigma)}=\frac{1}{b} \sum_{\sigma \in ! H} \chi^{(\sigma) n^{c(\sigma)}} .
$$

Since we have assumed $n$ large compared with $m$, we may equate coefficients in (1). One conclusion is immediate: $b=m ! / d$. Thus $H$ cannot be too small. If $m \leq 4$, the degrees of possible $K(T)$ which might be irreducible are easily computed and using (1), we easily obtain cases (c), (d), and (e) of the Theorem.

Suppose we are given a $K(T)$ which is a candidate for irreducibility. How do we find the tableau $Y$ to which it must correspond? We first describe a basis of $V_{\mathbf{X}}^{m}(H)$. Let $\Gamma_{m, n}$ be the $n^{m}$ sequences of length $m$ chosen from $\{1, \cdots, n\}$. If $\alpha=\left(\alpha_{1}, \cdots, \alpha_{m}\right)$ and $\beta=\left(\beta_{1}, \cdots, \beta_{m}\right) \in \Gamma_{m, n}$, we say $\alpha$ and $\beta$ are equivalent $(\bmod H)$ if there is a $\sigma \in H$ such that $\alpha_{\sigma(i)}=\beta_{i}, i=1, \cdots, m$, and write $\alpha^{\sigma}=\beta$. From each equivalence class thus defined, choose the element lowest in lexicographic order. Call this set of representatives $\Delta$. For each $a \in \Delta$, let $H_{\alpha}$ be the elements of $H$ which stabilize $\alpha$. Let $\bar{\Delta}=\left\{\alpha \in \Delta \mid \chi\left(H_{\alpha}\right) \equiv 1\right\}$. If $w_{1}, \cdots, w_{m} \in V$, write $T_{\mathbf{\chi}}^{H} w_{1} \otimes \cdots \otimes w_{m}=w_{1} * \cdots * w_{m}$. Let $v_{1}, \cdots, v_{m}$ be a basis of $V$. Then [5], $\left\{v_{a_{1}} * \ldots * v_{a_{m}} \mid a \in \bar{\Delta}\right\}$ is a basis of $V_{\mathbf{x}}^{m}(H)$.

To each $\alpha \in \bar{\Delta}$, associate a re-arrangement $\alpha^{0}$ of $a$ such that $\alpha_{1}^{0} \leq \cdots \leq \alpha_{m}^{0}$. From this set of $a^{0}$, pick the element $\gamma$ which is lowest in lexicographic order. (Of course, $\gamma$ may appear more than once.) Clearly, $\gamma$ will have the form $\gamma=$ $(1, \cdots, 1,2, \cdots, 2, \cdots, r, \cdots, r)$, where 1 occurs $m_{1}$ times $, \cdots, r$ occurs $m_{r}$ times, and $m_{1} \geq \cdots \geq m_{r}$

Lemma 2. Let $K(T)$ be irreducible. Let $\gamma$ be chosen as described above. Let $Y$ be the tableau with rows of lengths $m_{1}, \cdots, m_{r}$. Then $K(T)$ is equivalent to the representation of $\mathrm{GL}_{n}(C)$ corresponding to $Y$.

Proof. Let $T$ be a generic member of $\mathrm{GL}_{n}(C)$ with eigenvalues $x_{1}, \cdots, x_{n}$. Then $x_{a_{1}} \cdots x_{a_{m}}, \alpha \in \bar{\Delta}$, are the eigenvalues of $K(T)$. It follows from the choice of $\gamma$ that $x_{1}^{m_{1}} \cdots x_{r}^{m_{r}}$ is an eigenvalue of $K(T)$. Thus if $m_{1}^{\prime}, \cdots, m_{s}^{\prime}, m_{1}^{\prime} \geq \cdots \geq m_{s}^{\prime}$, were an other partition of $m$ which preceded $m_{1}, \ldots, m_{r}, x_{1}^{m_{1}^{\prime}} \ldots x_{s}^{m_{s}^{\prime}}$ cannot be an eigenvalue of $K(T)$. Let $T \rightarrow M(T)$ be the representation of $\mathrm{GL}_{n}(C)$ corresponding to $Y$. Recall that $S$ is the symmetrizer obtained from $Y$. If $w_{1}, \cdots, w_{m} \in V$, write $S w_{1} \otimes \cdots \otimes w_{m}=w_{1} \circ \cdots \circ w_{m}$. If $v_{1}, \cdots, v_{n}$ is a basis of $V$, there is a subset $\bar{\Delta}^{\prime} \subset \Gamma_{m, n}$ such that $\left\{v_{a_{1}} \circ \cdots \circ v_{a_{m}} \mid \alpha \in \bar{\Delta}^{\prime}\right\}$ is a basis of $S W$. It also follows that the eigenvalues of $M(T)$ will be $x_{\alpha_{1}} \cdots x_{a_{m}}, a \in \bar{\Delta}^{\prime}$. Let $v$ be the tensor $v_{1}$ $\otimes \cdots \otimes v_{1} \otimes \cdots \otimes v_{r} \otimes \cdots \otimes v_{r}$ where $v_{i}$ occurs $m_{i}$ times. The tableau $Y$ has the form 


$$
\begin{aligned}
& 1,2,3, \cdots, m_{1} \\
& Y=m_{1}+1, \quad m_{1}+m_{2} \text {, } \\
& \text {. } \\
& \text {. } \\
& \text {... } m
\end{aligned}
$$

so

$$
S v=\frac{d}{m !} \sum_{\sigma \in R ; \tau \in Q} \epsilon(\tau) P(\tau) P(\sigma) v=\frac{r d}{m !} \sum_{\tau \in Q} \epsilon(\tau) P(\tau) v
$$

where $r$ is the order of $R$. Clearly, the stabilizer $Q_{\gamma}$ of $\gamma$ in the column group of $Y$ is $\{1\}$, and thus $\Sigma_{\tau \in Q} \epsilon(\tau) P(\tau) v \neq 0$. Therefore, $M(T)$ has $x_{1}^{m_{1}} \ldots x_{r}^{m_{r}}$ as an eigenvalue.

Now let $m_{1}^{\prime} \geq \cdots \geq m_{s}^{\prime}$ be a partition of $m$ which precedes $m_{1}, \cdots, m_{r}$. Let $\beta \in \Gamma_{m, n}$ be any sequence in which $i$ occurs $m_{i}^{\prime}$ times, $i=1, \ldots, s$. We will show that $S v_{\beta_{1}} \otimes \ldots \otimes v_{\beta_{m}}=0$. Suppose that $i$ appears in positions $t_{i, 1}, \cdots, t_{i, m_{i}^{\prime}} \cdot$ Let $Y_{1}$ be the tableau with row lengths $m_{1}^{\prime}, \ldots, m_{s}^{\prime}$ such that the $i$ th row consists of the integers $t_{i, 1}, \cdots, t_{i, m_{i}^{\prime}}$. Let $R_{1}$ and $Q_{1}$ be the row and column groups of $Y_{1}$. Clearly (writing $v_{\beta}=v_{\beta_{1}}^{i} \otimes \cdots \otimes v_{\beta_{m}}$ ),

$$
\sum_{\sigma \in R} P(\sigma) v_{\beta}=r_{1} v_{\beta}
$$

where $r_{1}$ is the order of $R_{1}$. Thus,

$$
\begin{aligned}
S v_{\beta} & =\frac{d}{r_{1} m !} \sum_{\tau \in Q} \sum_{\sigma \in R} \epsilon(\tau) P(\tau \sigma) \sum_{\rho \in R_{1}} P(\rho) v_{\beta} \\
& =\frac{d}{r_{1} m !} \sum_{\sigma \in R} \sum_{\tau \in Q} \epsilon(\tau) P\left(\sigma \tau_{\sigma}\right) \sum_{\rho \in R_{1}} P(\rho) v_{\beta},
\end{aligned}
$$

where $\tau_{\sigma}$ is the element in the column group of the tableau $\sigma Y$ such that $\tau_{\sigma}=$ $\sigma^{-1} \tau \sigma[1$, p. 106]. Thus, (2) becomes

$$
\frac{d}{r_{1} m !} \sum_{\sigma \in R} P(\sigma) \sum_{\tau \in Q} \epsilon\left(\tau_{\sigma}\right) P\left(\tau_{\sigma}\right) \sum_{\rho \in R_{1}} P(\rho) v_{\beta} .
$$

Now $Y_{1}$ dominates $Y$, and from [1, p. 107] we know that the column symmetrizer from a tableau with the same shape as $Y$ times the row symmetrizer from any tableau with shape dominating $Y$ is zero. Thus (3) is zero. Thus, if $Y_{1}$ is any tableau which dominates $Y$, the representation $M_{1}(T)$ corresponding to $Y_{1}$ has eigenvalues which do not occur in $K(T)$; if $Y$ dominates $Y_{1}, x_{1}^{m_{1}} \ldots x_{r}^{m_{r}}$ is not an eigenvalue of $M_{1}(T)$. Lemma 2 is proved. 
We use Lemma 2 and the following three results to eliminate groups which have no 2-cycles or 3-cycles. Let $\Omega$ be the elements in $H$ which are 3 -cycles or a product of two disjoint 2-cycles.

Lemma 3. Let $H$ be a transitive subgroup of $S_{m}$ baving no 2-cycles or 3-cycles. Let $H$ bave $r$ blocks of size $k$, where $k>1$ is as small as possible. Then if $k \geq$ 9, $\Omega$ is empty.

Proof. Assume (12)(34) $\in H$. As long as $k \geq 5$, we may assume $1,2,3,4$ are all in the same block. We will use the fact that if $\sigma, \tau \in S_{m}$ and if the elements moved by $\sigma$ and the elements moved by $\tau$ have only one element in common, then $\sigma \tau \sigma^{-1} \tau^{-1}$ is a 3 -cycle. We consider two cases. First suppose $(13)(24)$ is also in $H$. Now $\{1,2,3,4\}$ is not a block, so there is an element $\phi$ of $H$ sending 1 to 5 and sending 2 to 1,3 or 4 . By conjugating $\phi$ with (12)(34), we produce $(\phi(2) 5)(\phi(3) \phi(4))$. Since $(13)(24)$ and $(14)(23) \in H$, we can produce an element of the form $(15)(\psi(3) \psi(4))$. Now exactly one of $\psi(3)$ and $\psi(4)$ is not in $\{2,3,4\}$; otherwise we could produce a 3 -cycle in $H$. Thus we may assume $(15)(26) \in H$, so $H$ contains the group (12)(34), (13)(24), (15)(26). Now $\{1,2, \cdots, 6\}$ is not a block of $H$, so we can produce an element $\phi \in H$ of the form $(17)(\phi(3) \phi(4))$. Because the roles of 3 and 4 are interchangeable, the only possibilities for $(\phi(3) \phi(4))$ are (35), (36) or (28). Thus if $H_{1}=\langle(12)(34),(13)(24),(15)(26)\rangle, H$ must contain one of the following groups: $\left\langle H_{1},(17)(35)\right\rangle,\left\langle H_{1},(17)(36)\right\rangle,\left\langle H_{1},(17)(28)\right\rangle$. Now proceed in the same manner. When an element $\sigma$ of the form $(19)(\phi(3) \phi(4))$ is produced, we have for every possibility for $\phi(3), \phi(4)$, an element whose commutator with $\sigma$ is a 3-cycle. In the second case, we assume (13)(24) and (14)(23) are not in $H$. Now $\{1,2,3,4\}$ is not a block, so we are lead in a similar manner to assume that one of the following elements is also in $H:(15)(23)$, or $(15)(36)$. (Note that $(15)(26)$ is not a possibility, because (12)(56) would then be in $H$ and we could return to case 1.) We now use the same procedure as in case 1 to obtain the result.

Lemma 4. Let $Y$ be a tableau with rows of length $m_{1} \geq \cdots \geq m_{r}, \Sigma m_{i}=m$. If $m_{1}>m / 2$, then the coefficient of $n^{m-1}$ in (1) is $\geq 0$, with equality if and only if $m_{2}=\cdots=m_{r}=1$ and $m_{1}=m+1 / 2$.

Proof. Use induction on $m$. Verification is easy for small $m$. If $m_{2}=\cdots=$ $m_{r}=1$, the lemma is clear, so assume $m_{2} \geq 2$. Let $Y_{1}$ be the tableau obtained from $Y$ by deleting the last positions in rows 1 and $r$. Then $Y_{1}$ satisfies the inductive hypothesis, so the coefficient $\lambda$ of $n^{m-3}$ corresponding to $Y_{1}$ is nonnegative. The coefficient of $n^{m-1}$ corresponding to $Y$ is $\lambda+\left(m_{1}-1\right)+\left(m_{r}-1\right)-r$. Since $m_{2}$ $\geq 2, r \leq m_{1}-1$. Thus $\lambda+\left(m_{1}-1\right)+\left(m_{r}-1\right)-r \geq \lambda$. But $\lambda=0$ only if $m_{2}=2$ and $r=2$. In this case $m_{r}=2$ and thus the coefficient of $n^{m-1}$ is positive. 
Lemma 5. Let $K(T)$ be irreducible with corresponding tableau $Y$. If $m \geq 5$, and $H$ bas no 2-cycles, then the coefficient of $n^{m-2}$ in $\Sigma_{\sigma \in R ; \tau \in Q} \epsilon(\tau) n^{c(\tau \sigma)}$ is less then $-m$.

Proof. If $Y$ has row lengths $m_{1} \geq \cdots \geq m_{r}$ and column lengths $n_{1} \geq \cdots \geq n_{s}$, then since $H$ has no transpositions,

$$
\sum_{i=1}^{r}\left(\begin{array}{c}
m_{i} \\
2
\end{array}\right)=\sum_{i=1}^{s}\left(\begin{array}{c}
n_{i} \\
2
\end{array}\right)
$$

The coefficient of $n^{m-2}$ is

$$
\sum_{i=1}^{r} 3\left(\begin{array}{c}
m_{i} \\
4
\end{array}\right)+2\left(\begin{array}{c}
m_{i} \\
3
\end{array}\right)+\sum_{i=1}^{s} 3\left(\begin{array}{c}
n_{i} \\
4
\end{array}\right)+2\left(\begin{array}{c}
n_{i} \\
3
\end{array}\right)-\sum_{i=1}^{r} \sum_{j=1}^{s}\left(\begin{array}{c}
m_{i} \\
2
\end{array}\right)\left(\begin{array}{c}
n_{j} \\
2
\end{array}\right)
$$

This expression is obtained by observing that a 3-cycle or a product of two disjoint 2-cycles is of the form $\tau \sigma, \tau \in Q, \sigma \in R$, if and only if both $\sigma$ and $\tau$ are 2-cycles or one of $\sigma$ and $r$ is the identity, and the other is a 3-cycle or product of two disjoint 2-cycles. Using (4), write (5) as

$$
\begin{aligned}
& \sum_{i=1}^{r}\left(\begin{array}{c}
m_{i} \\
4
\end{array}\right)+2\left(\begin{array}{c}
m_{i}+1 \\
4
\end{array}\right)+\sum_{i=1}^{s}\left(\begin{array}{c}
n_{i} \\
4
\end{array}\right)+2\left(\begin{array}{c}
n_{i}+1 \\
4
\end{array}\right)-\left[\sum_{i=1}^{r}\left(\begin{array}{c}
m_{i} \\
2
\end{array}\right)\right]^{2} \\
& <\frac{1}{2}\left[\sum_{i=1}^{r}\left(\begin{array}{c}
m_{i} \\
2
\end{array}\right)^{2}+\sum_{i=1}^{s}\left(\begin{array}{c}
n_{i} \\
2
\end{array}\right)^{2}\right]-\left[\sum_{i=1}^{r}\left(\begin{array}{c}
m_{i} \\
2
\end{array}\right)\right]^{2} \\
& =\frac{1}{2}\left[\left(\sum_{i=1}^{r}\left(\begin{array}{c}
m_{i} \\
2
\end{array}\right)\right)^{2}+\left(\sum_{i=1}^{s}\left(\begin{array}{c}
n_{i} \\
2
\end{array}\right)\right)^{2}-2 \sum_{i \neq j}\left(\begin{array}{c}
m_{i} \\
2
\end{array}\right)\left(\begin{array}{c}
m_{j} \\
2
\end{array}\right)\right. \\
& \left.-2 \sum_{i \neq j}\left(\begin{array}{c}
n_{i} \\
2
\end{array}\right)\left(\begin{array}{c}
n_{j} \\
2
\end{array}\right)\right]-\left[\sum_{i=1}^{r}\left(\begin{array}{c}
m_{i} \\
2
\end{array}\right)\right]^{2} \\
& =-\sum_{i \neq j}\left(\begin{array}{c}
m_{i} \\
2
\end{array}\right)\left(\begin{array}{c}
m_{j} \\
2
\end{array}\right)-\sum_{i \neq j}\left(\begin{array}{c}
n_{i} \\
2
\end{array}\right)\left(\begin{array}{c}
n_{j} \\
2
\end{array}\right) \text {. }
\end{aligned}
$$

With expression (6), we may assume that $\left(m_{1}, \cdots, m_{r}\right)$ precedes $\left(n_{1}, \cdots, n_{s}\right)$. Thus $m_{1} \geq \sqrt{m}$. If $m_{2} \geq 3, n_{1}, n_{2}, n_{3}$ are all at least 2 . Thus $(6)$ is no larger than

$$
-\left(\begin{array}{c}
m_{1} \\
2
\end{array}\right)\left(\begin{array}{c}
m_{2} \\
2
\end{array}\right)-3 \leq-\frac{3}{2} \sqrt{m}(\sqrt{m}-1)-3<-m .
$$


If $m_{2}=2$, we must have $m_{3}=\cdots=m_{r}=1$. From (4), we have $n_{1}=m_{1}$ and $Y$ is symmetric. Thus $2 m_{1}=m$ and (6) becomes

$$
-2\left(\begin{array}{c}
m_{1} \\
2
\end{array}\right)=-m\left(\frac{m}{2}-1\right)<-m
$$

for $m \geq 5$. Finally, if $m_{2}=1$, we verify $Y$ is symmetric with $m_{1}=(m+1) / 2$. Then we write (5) as

$$
6\left(\begin{array}{c}
m_{1} \\
4
\end{array}\right)+4\left(\begin{array}{c}
m_{1} \\
3
\end{array}\right)-\left(\begin{array}{c}
m_{1} \\
2
\end{array}\right)^{2}
$$

and this is less than $-m$ for $m \geq 6$.

If $m=5$, we need 5 elements in $\Omega$ for equality to hold in Lemma 5 . Thus, to within conjugation, $H$ must contain the group $G=\langle(12)(34),(13)(25)\rangle$, which is of order 10. The degree $d$ of the corresponding representation of $S_{5}$ is 6: thus, $b=$ 20. But no subgroup of $S_{5}$ of order 20 contains $G$.

Now suppose $H$ has no 2-cycles or 3 -cycles. Let $H$ divide $\{1, \cdots, m\}$ into $r$ blocks of length $k$, where $k>1$ is as small as possible. Since $\chi$ is linear, we see by equating the coefficients of $n^{m-2}$ in (1) that there must be more than $m$ elements in $\Omega$ (the coefficient of $n^{m-2}$ is $\Sigma_{\sigma \in \Omega} \chi^{(\sigma))}$ ). By Lemma 3, we assume $k \leq 8$. Note that if $5 \leq k \leq 8$, we may assume that (12)(34) $\in H$ and $\{1,2,3,4\}$ is part of a block. First we eliminate the case $k=8$. Consider the sequence

$$
a=(1,1,2,1,1,3,4,5 ; 1,1,2,1,1,3,4,6 ; 1,1,2,1,1,3,4,7 ; \ldots) .
$$

We assert that $\alpha$ is equivalent to a member of $\bar{\Delta}$; in fact, $H_{a}=\{(1)\}$. Since $H$ is transitive, we also assume that $(910)\left(\begin{array}{ll}11 & 12\end{array}\right)$, etc., are in $H$. Thus, $(12)(45)$, (9 10)(12 13), etc. are not in $H$. It is possible, however, that something like $(12)(45)(910)(1213)$ or $(124)(91012) \in H$, and we must eliminate these cases. If $H_{a} \neq\{1\}$, let $\sigma \in H_{a}$. Then $\sigma$ is of order at most 12. If $\sigma$ contains 3-cycles, look at $\tau=\sigma^{4}$; it is a product of 3-cycles only. Assume the number of 3-cycles is minimal in $H_{a}$. Thus $\tau$ is of the form $\rho_{1} \rho_{2}$, where $\rho_{1}$ is a 3 -cycle in $\{1,2,4,5\}$ and $\rho_{2}$ is a product of 3 -cycles fixing $\{1,2,4,5\}$. Suppose $\rho_{1}=(124)$. If $(13)(24)$ is also in $H$, we may assume, as in Lemma 3 that $(15)(26) \in H$. Then $(15)(26) \rho_{1} \rho_{2}=$ $(16245) \rho_{2}$. Raise this to the 5 th power to contradict the minimality of 3-cycles. If $(13)(24) \notin H$, we may assume $(15)(36)$ or $(13)(25) \in H$. Now $(15)(36) \rho_{1} \rho_{2}=$ $(1245)(36) \rho_{2}$ and (13)(25) $\rho_{1} \rho_{2}=(15243) \rho_{2}$. Raising these to suitable powers also yields a contradiction. Similarly, if $\rho_{1}=(125)$, (145) or (245), we use the same procedure. If $\sigma$ has no 3-cycles, write $\sigma$ as $\rho_{1} \rho_{2}$, where $\rho_{1}$ permutes $\{1,2,4,5\}$. Again, similar procedures yield the result, and we have $H_{\alpha}=\{1\}$. 
It follows that in $Y, m_{1}=m / 2$. (If $m_{1}>m / 2$, Lemma 4 yields the existence of a transposition in $H$.) Moreover, $m_{2}+m_{3}+m_{4} \geq 3 m / 8$. Thus

$$
\left(\begin{array}{c}
m_{2} \\
2
\end{array}\right)+\left(\begin{array}{c}
m_{3} \\
2
\end{array}\right)+\left(\begin{array}{c}
m_{4} \\
2
\end{array}\right) \geq 3\left(\begin{array}{c}
m / 8 \\
2
\end{array}\right) .
$$

Let $r_{i}=\max \left\{n_{i}-3,0\right\}$. Thus $\Sigma r_{i} \leq m / 8$. We compute

$$
\begin{aligned}
\sum\left(\begin{array}{c}
m_{i} \\
2
\end{array}\right)-\sum\left(\begin{array}{c}
n_{i} \\
2
\end{array}\right) & >\left(\begin{array}{c}
m / 2 \\
2
\end{array}\right)+3\left(\begin{array}{c}
m / 8 \\
2
\end{array}\right)-\sum\left(\begin{array}{c}
r_{i} \\
2
\end{array}\right)-\left(\begin{array}{l}
3 \\
2
\end{array}\right) \sum r_{i}-\left(\begin{array}{l}
3 \\
2
\end{array}\right) \frac{m}{2} \\
& \geq\left(\begin{array}{c}
m / 2 \\
2
\end{array}\right)+3\left(\begin{array}{c}
m / 8 \\
2
\end{array}\right)-\left(\begin{array}{c}
m / 8 \\
2
\end{array}\right)-3 m / 8-3 m / 2 .
\end{aligned}
$$

This is positive for $m \geq 16$. If $m=8$, the only tableau with $m_{1}=4$ and (1) holding is $m_{1}=4, m_{2}=2, m_{3}=m_{4}=1$. This tableau corresponds to a representation of $S_{8}$ of degree 90 , and hence $b=2^{6} \cdot 7$. Assume $(12)(34) \in H$. If (13)(24) and (14)(23) $\epsilon H$, we see that the number of elements conjugate to (12)(34) is divisible by 3 , a contradiction. If $(13)(24) \notin H$, then, as previously shown, we may assume $(15)(36)$ or $(15)(23) \in H$. Now $(12)(34)(15)(36)$ has order 3 and (12)(34) and $(15)(23)$ generate a group of order 10 . Both cases are impossible.

If $k=7$, we can similarly show that the sequence $\alpha=(1,1,2,1,1,3,4 ; 1,1$, $2,1,1,3,5 ; \cdots)$ has $\{1\}$ as its stabilizer. If $k=6$, we also show that $\alpha=(1,1$, $2,1,1,3 ; 1,1,2,1,1,4 ; \cdots)$ has $H_{a}=\{1\}$. In both cases, apply Lemma 4 .

If $k=5$, and (12)(34) $\in H$, then we may assume that (13)(25), (14)(35), (15)(24), (23)(45) are in $H$. Thus, the stabilizer of $(1,1,1,2,3 ; 1,1,1,2,4 ; \cdots)$ is $\{1\}$. Now use Lemma 4.

Let $k=4,(12)(34) \in H, \chi(12)(34)=-1$. Suppose $\{1,2,3,4\}$ is a block. Then the only possible elements of $\Omega$ which do not fix 1 and 2 are of the form (13)(24), $(14)(23)$ or $(12)(i j), i, j \geq 5$. Since $\chi((12)(i j))=-\chi((34)(i j))$, we cannot have (12)(ij) and (13)(24) in $H$. Thus, if (13)(24) $\in H, \Sigma_{\sigma \in \Omega} \chi(\sigma) \geq-r$. If (12)(ij) $\in H$, then $\Sigma_{\sigma \subset \Omega} \chi(\sigma)=0$. If $(12)(i j),(13)(24) \notin H$, then $\Sigma_{\sigma \in \Omega} \chi(\sigma) \geq-r$. In all cases, we apply Lemma 5.

If $\{1,2,3,4\}$ is not a block, we may assume the blocks of $H$ are $\{1,2,5,6\}$, $\{3,4,7,8\},\{9,10,11,12\}, \ldots$. Now $(12)(56) \notin H$; otherwise, we could return to the first case. Thus, any other elements in $\Omega$ which move 1 or 2 must be of the form $(12)(910)$ or $(15)(37)$. These two possibilities cannot both occur. If $(12)(910)$ $\epsilon H$, we handle this as above. If $(15)(37) \epsilon H$, the possible members of $\Omega$ which act on $\{1, \cdots, 8\}$ are $(12)(34),(15)(37),(25)(47),(16)(38),(26)(48),(56)(78)$. All other members of $\Omega$ fix each of $\{1, \cdots, 8\}$. Since $H$ is transitive, $\Sigma_{\sigma \in \Omega} \chi(\sigma)>$ - 3m/4. Apply Lemma 4. 
If $k=3$, we may assume the blocks of $H$ are $\{1,2,5\},\{3,4,6\},\{7,8,9\}, \cdots$. Since $\{1,2\}$ is not a block, there is a $\sigma \in H$ mapping $1 \rightarrow 5$ and $2 \rightarrow 1$. Thus $(15)(i j) \in H$ and we may assume (to avoid 3-cycles) that $(i j)=(36)$ or $(46)$. Thus assume that $(12)(34),(15)(36),(25)(46) \in H$. It is easy to check that all other elements of $\Omega$ fix each of $1, \cdots, 6$. We can again use Lemma 5 .

If $k=2$, we see that the stabilizer of $(1,1 ; 1,2 ; 1,3 ; \cdots ; 1, r)$ is $\{1\}$. If this sequence does not correspond to the tableau $Y$, we can use Lemma 4. Otherwise, $Y$ satisfies $m_{1}=r+1, m_{2}=\cdots=m_{r-1}=1$. Then $d=\left(\begin{array}{c}2 r-1 \\ r\end{array}\right)$. Now $H$ is properly contained in the Kranz product of $S_{2}$ with $S_{r}$ and hence $m ! / d=b<2^{r} r$. This is impossible unless $r=1$. But then $H=S_{2}$ and we are done.

We now assume $H$ has a 3 -cycle but no 2-cycles. A primitive group with a 2 cycle or 3-cycle is $A_{m}$ or $S_{m}$, so assume $H$ is not primitive. Since $H$ has a 3 cycle, there is a copy of $A_{3}$ in $H$. Let $k$ be the largest integer such that $H$ has a copy of $A_{k}$. We may assume that this group acts on $\{1, \cdots, k\}$. Then $k \mid m$ and if $r k=m, H$ has $r$ blocks of length $k$. Moreover, $H$ contains the direct product of $r$ copies of $A_{k}$, because $H$ is transitive. Note that $b<(k !)^{r} r$ !. If $k=4$, a simple induction shows that $b<\sqrt{m !}$ for $r \geq 5$ and if $k=3$, we can show that $b<\sqrt{m !}$ for $r \geq 4$. Then $m ! / b=d>\sqrt{m !}$. This is absurd because $d$ is the degree of an irreducible representation of $S_{m}$.

Suppose $k=3$. If $\chi\left(X_{1}^{r} A_{k}\right) \equiv 1$, then $(1,1,1,1,2,3)$ is equivalent to a member of $\bar{\Delta}$ when $r=2$ and $(1,1,1,1,2,3,1,2,4)$ is equivalent to a member of $\bar{\Delta}$ when $r=3$. In both cases, apply Lemma 4 . If $\chi\left(X_{1}^{r} A_{k}\right) \not \equiv 1$, then for $r=2$ and 3 respectively, the sequences $(1,1,2,1,1,3)$ and $(1,1,2,1,1,3,1,2,3)$ are equivalent to members of $\bar{\Delta}$ unless $(12)(45) \in H$ and $\chi((12)(45))=-1$. But $\chi((123))=\eta$, a cube root of 1. Thus $\chi((12)(45)(123))=\chi((23)(45))=-\eta$, a contradiction. Again we can apply Lemma 4 .

Suppose $k=4$, and $\chi\left(\mathrm{X}_{1}^{r} A_{k}\right) \equiv 1$. If $r=2,(1,1,1,1,1,2,3,4)$ is in $\bar{\Delta}$ we apply Lemma 4 . If $r=3$, the sequence $(1,1,1,1 ; 1,1,2,3 ; 1,1,2,4)$ is equivalent to a member of $\bar{\Delta}$ unless we have $\sigma(56) \in H$ and $\chi(\sigma(56)) \neq 1$ where $\sigma$ is an odd permutation of $\{1,2,3,4\}$. Then $(12)(56) \in H$ and $\chi((12)(56))=-1$. If this is so, consider (12)(9 10); if it is in $H$ and $\chi((12)(910))=-1$, then $\chi((56)(910))=1$ and we have $(1,2,3,4 ; 1,1,1,1 ; 1,1,1,2)$ equivalent to a member of $\bar{\Delta}$. If

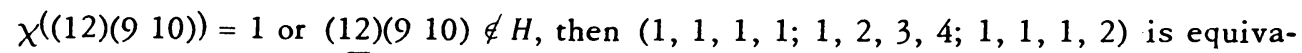
lent to an element of $\bar{\Delta}$. In all cases, apply Lemma 4 . If $r=4$, we similarly assume that $(1,1,1,1 ; 1,1,1,2 ; 1,2,3,4 ; 1,2,3,5)$ is equivalent to a member of $\bar{\Delta}$. Apply Lemma 4.

If $k=4$ and $\chi\left(X_{1}^{r} A_{k}\right) \equiv 1$, then $\chi$ is a primitive cube root of 1 on any 3 -cycle in $H$ and $\chi((12)(34))$ and all of its conjugates is 1 . As with the case $k=3$, we can verify that the sequence $(1,1,2,2 ; 1,1,2,3 ; 1,1,2,4 ; 1,1,2,5)$ is equivalent to an element of $\bar{\Delta}$ when $r=4$. Thus the tableau $Y$ to which $K(T)$ corresponds 
has first row length 8 and second row length at least 5. It is easily shown that any tableau satisfying this also satisfies

$$
\sum\left(\begin{array}{c}
m_{i} \\
2
\end{array}\right)-\sum\left(\begin{array}{c}
n_{i} \\
2
\end{array}\right)>0
$$

contradicting the assumption that $H$ has no transpositions. The cases $r=3,2$ are disposed in similar fashion.

We now assume $k \geq 5$ and thus $\chi\left(X_{1}^{r} A_{k}\right) \equiv 1$. Let $\alpha$ be an element of $\bar{\Delta}$ which has $m_{1} 1$ 's, $m_{2} 2$ 's, $\cdots$, i.e., a determines the shape of the tableau $Y$. Write

$$
\alpha=\left(a_{11}, \cdots, \alpha_{1 k} ; \alpha_{21}, \cdots, \alpha_{2 k} ; \cdots ; \alpha_{r 1}, \cdots, \alpha_{r k}\right) .
$$

Suppose that each of the sets $\left\{\alpha_{i 1}, \cdots, \alpha_{i k}\right\}, i=1, \cdots, s$ has a repetition, and that for $i=s+1, \cdots, r, \alpha_{i 1}, \cdots, \alpha_{i k}$ are all distinct. Then the sequence

$$
\begin{aligned}
\beta=(1, \cdots, 1 ; 1, \cdots, 1,2 ; \cdots ; 1, \cdots, 1, s ; 1,2, \cdots, k \\
1,2, \cdots, k-1, k+1 ; \cdots ; 1,2, \cdots, k-1, r-s+1)
\end{aligned}
$$

is equivalent to a member of $\bar{\Delta}$. The sequence $\alpha$ may have more 1 's than $\beta$ and more $k$ 's than $\beta$, but $2, \cdots, k-1$ each will occur in $\alpha$ at least $r-s$ times. If $s \geq r / 2$, we could apply Lemma 4 , so assume that $2, \cdots, k-1$ each occur in $\alpha$ at least $r / 2$ times. Thus the tableau $Y$ has at least $r / 2$ columns of length at least $k-1$.

Recall that $\Omega$ consists of the 3-cycles and products of 2 disjoint 2-cycles in $H$. The cardinality of $\Omega \subset X_{1}^{r} A_{k}$ is $2 r\left(\begin{array}{l}k \\ 3\end{array}\right)+3 r\left({ }_{4}^{k}\right)$. There are also elements of the form $(12)(k+1, k+2)$, which are not in $\mathrm{X}_{1}^{r} A_{k}$. If $(12)(k+1, k+2)$ and $(12)(2 k+1,2 k+2)$ are in $H$ and $\chi=-1$ on both, then $\chi((k+1, k+2)(2 k+1,2 k+2))$ $=1$. Using this, we verify that

$$
\sum_{\sigma \in \boldsymbol{\Omega}} \chi(\sigma) \geq 2 r\left(\begin{array}{l}
k \\
2
\end{array}\right)+3 r\left(\begin{array}{l}
k \\
4
\end{array}\right)-\left[\frac{r}{2}\right]\left(\begin{array}{l}
k \\
2
\end{array}\right)^{2} .
$$

From (6) in Lemma 5, we have

$$
\begin{aligned}
& \sum_{\sigma \in \Omega} \chi(\sigma)<-\sum_{i \neq j}\left(\begin{array}{c}
m_{i} \\
2
\end{array}\right)\left(\begin{array}{c}
m_{j} \\
2
\end{array}\right)-\sum_{i \neq j}\left(\begin{array}{c}
n_{i} \\
2
\end{array}\right)\left(\begin{array}{l}
n_{j} \\
2
\end{array}\right) \\
& \leq\left(\begin{array}{c}
k-1 \\
2
\end{array}\right)\left(\begin{array}{c}
{[1 / 2 r]} \\
2
\end{array}\right)^{2}-\left(\begin{array}{c}
{[1 / 2 r} \\
2
\end{array}\right)\left(\begin{array}{c}
k-1 \\
2
\end{array}\right) \text {. }
\end{aligned}
$$

One shows for $r, k \geq 4$ that (8) is less than (7), a contradiction. If $r=3$, we cannot have all three of $(12)(k+1, k+2),(12)(2 k+1,2 k+2)$ and $(k+1, k+2)(2 k+1,2 k+2)$ 
in $H$ with $\chi$ taking the value -1 . So suppose $\chi((12)(k+1, k+2))=1$ or $(12)(k+1, k+2) \notin H$. Then $(1, \ldots, 1 ; 1,1, \ldots, 1,2 ; 123, \ldots, k)$ is in $\bar{\Delta}$ and we use Lemma 4. If $r=2$, either $(1, \cdots, 1 ; 1, \cdots, 1,2) \in \bar{\Delta}$ or $(1,111,1 ; 1,2, \cdots, k)$ $\epsilon \bar{\Delta}$. In the first case use Lemma 4. In the second case, since $H$ has no 2-cycles, $b<(k !)^{2}$. But $(1, \cdots, 1 ; 1,2, \cdots, k)$ is clearly the sequence which produces $Y$. Thus $Y$ satisfies $m_{1}=k+1, m_{2}=\cdots=m_{k}=1$. The degree $d$ of the representation of $S_{m}$ corresponding to $Y$ is $\left(\begin{array}{c}2 k-1 \\ k\end{array}\right)$. But $b<(k !)^{2}$ implies $d>\left(\begin{array}{c}2 k \\ k\end{array}\right)$, a contradiction.

We have now eliminated all cases where $r \geq 2$. Of course, if $r=1, H=A_{k}$ and we use Lemma 1.

Now assume $H$ contains a transposition. As before, we conclude that $H$ contains $X_{l}^{r} S_{k}$, where the different copies of $S_{k}$ are all conjugate.

There are two possibilities:

Case 1. $\chi\left(X_{1}^{r} s_{k}\right)=X_{1}^{r} \epsilon$. Then the sequence $(1, \cdots, k ; 1, \cdots, k-1, k+1$; $\cdots ; 1, \cdots, k-1, k+r-1)$ is in $\bar{\Delta}$. This sequence may not be the sequence $\gamma$ corresponding to $Y$, but clearly, $1, \cdots, k-1$ each occur exactly $r$ times in $\gamma$. Let the row lengths of $Y$ be $r, \cdots, r, l_{1}, \cdots, l_{q}, \Sigma l_{i}=r_{\text {o }}$ Let the column lengths of $Y$ be $k-1+s_{i}, i=1, \cdots, r, \Sigma s_{i}=r$. Then

$$
\begin{aligned}
\sum\left(\begin{array}{c}
m_{i} \\
2
\end{array}\right)-\sum\left(\begin{array}{c}
n_{i} \\
2
\end{array}\right) & =(k-1)\left(\begin{array}{l}
r \\
2
\end{array}\right)+\sum\left(\begin{array}{c}
l_{i} \\
2
\end{array}\right)-r\left(\begin{array}{c}
k-1 \\
2
\end{array}\right)-\sum\left(\begin{array}{c}
s_{i} \\
2
\end{array}\right)-\sum(k-1) s_{i}, \\
& =(k-1)\left(\begin{array}{l}
r \\
2
\end{array}\right)+\sum\left(\begin{array}{c}
l_{i} \\
2
\end{array}\right)-r\left(\begin{array}{c}
k-1 \\
2
\end{array}\right)-\sum\left(\begin{array}{c}
s_{i} \\
2
\end{array}\right)-r(k-1), \\
& \geq(k-1)\left(\begin{array}{l}
r \\
2
\end{array}\right)-r\left(\begin{array}{c}
k-1 \\
2
\end{array}\right)-\left(\begin{array}{l}
r \\
2
\end{array}\right)-r(k-1) .
\end{aligned}
$$

Now

$$
\sum_{\sigma=2-\text { cycle }} \chi(\sigma)=-r\left(\begin{array}{l}
k \\
2
\end{array}\right) .
$$

One verifies that (9) is larger than $-r\left(\frac{k}{2}\right)$ unless $r, k \leq 2$. But $r k=m \geq 5$.

Case 2. $\chi\left(X_{1}^{r} s_{k}\right) \equiv 1$. Then $(1, \ldots, 1 ; 1, \ldots, 2 ; 1, \ldots, 1,3 ; \cdots, 1, \cdots, 1, r)$

is equivalent to a member of $\bar{\Delta}$. Thus, in $Y, m_{1} \geq r(k-1)+1$. Let $r_{i}=n_{i}-1$. Then

$$
\begin{aligned}
\sum\left(\begin{array}{c}
m_{i} \\
2
\end{array}\right)-\left(\begin{array}{c}
n_{i} \\
2
\end{array}\right) & \geq\left(\begin{array}{c}
m-r+1 \\
2
\end{array}\right)-\left(\begin{array}{c}
r-1 \\
2
\end{array}\right)-\sum r_{i} \\
& =\left(\begin{array}{c}
m-r+1 \\
2
\end{array}\right)-\left(\begin{array}{c}
r-1 \\
2
\end{array}\right)-(r-1)=\left(\begin{array}{c}
m-r+1 \\
2
\end{array}\right)-\left(\begin{array}{l}
r \\
2
\end{array}\right) .
\end{aligned}
$$


Now

$$
\sum_{\sigma=2-\text { cycle }} \chi(\sigma)=r\left(\begin{array}{l}
k \\
2
\end{array}\right) .
$$

Now if $r \geq 2,(10)$ is larger than $r\left(\begin{array}{l}k \\ 2\end{array}\right)$ unless $k=2$. If $k=2$, then for equality to hold in (10), $Y$ must satisfy $m_{1}=r+1, m_{2}, \cdots, m_{r-1}=1$. Then $d=\left(\begin{array}{c}2 r-1 \\ r\end{array}\right)$. Now $H$ is contained in the Kranz product of $S$ with $S_{r}$ and hence $b \leq 2^{r} r$. Thus

$$
\frac{(2 r) !}{2^{r} r !} \leq \frac{(2 r-1) !}{r !(r-1) !} \text {. }
$$

Equality holds only when $r=2$. This forces $m=4$. But $m \geq 5$. Thus $r=1$ and $H=S_{m}$. Use Lemma 1 to finish the proof of the Theorem.

Corollary. If $(m, n, H, \chi)$ satisfy the conditions of the Theorem, then $\operatorname{Hom}_{C}\left(V_{x}^{m}(H), V_{x}^{m}(H)\right)$ is spanned by elements of the form $K(T), T \in \mathrm{GL}_{n}(C)$.

\section{REFERENCES}

1. H. Boerner, Darstellungen von Gruppen mit Berücksichtigung der Bedürfnisse der modernen Physik, Springer, Berlin, 1955; English transl., North-Holland, Amsterdam; Interscience, New York, 1963. MR 17, 710; MR $26 \# 6272$. Calif.

2. R. Freese, Private communication, California Institute of Technology, Pasadena,

3. M. Marcus, Lengths of tensors, Proc. Sympos. Inequalities (Wright-Patterson Air Force Base, Ohio, 1965), Academic Press, N ew York, 1967, pp. 163-176. MR 36 \#2634.

4. - The Hadamard theorem for permanents, Proc. Amer. Math. Soc. 15 (1964), 967-973. MR $29 \# 5845$.

5. M. Marcus and H. Minc, Generalized matrix functions, Trans. Amer. Math. Soc. 116 (1965), 316-329. MR 33 \#2655.

6. M. Marcus and M. Newman, Inequalities for the permanent function, Ann. of Math. (2) 75 (1962), 47-62. MR 25 \#96.

7. R. Merris and S. Pierce, A class of representations of the full linear group, J. Algebra 17 (1971), 346-351.

8. D. Passman, Permutation groups, Benjamin, New York, 1968. MR 38 \#5908.

9. I. Schur, Über endliche Gruppen und Hermitesche Formen, Math. Z. 1 (1918), 184-207.

10. H. Weyl, Der Zusammenhang zwischen der symmetrischen und der linearen Gruppe, Ann. of Math. 30 (1929), 499-516.

11. H. Wielandt, Finite permutation groups, Lectures, University of Tübingen, 1954/55; English transl,, Academic Press, New York, 1964. MR 32 \#1252.

12. S. G. Williamson, Operator theoretic invariants and the enumeration theory of Pólya and de Bruijn, J. Combinatorial Theory 8 (1970), 162-169. MR 40 \#5461. 